\title{
Ge-Ti (Germanium-Titanium)
}

\section{H. Okamoto}

The complete Ge-Ti phase diagram in [Massalski2] was redrawn from [1989Rud], which superseded a partial phase diagram (60-100 at.\% Ti) evaluated by [1987Mur].

[2011Liu] obtained the Ge-Ti phase diagram shown in Fig. 1 by thermodynamic calculation. Experimental phase boundary data reported by [1989Rud], reviewed by [1987Mur], and observed by the authors themselves were used as the basis of the thermodynamic model.

Table 1 shows Ge-Ti crystal structure data shown in [Massalski2] with modifications for consistency with Fig. 1.

\section{References}

1987Mur: J.L. Murray, The Ge-Ti (Germanium-Titanium) System, Phase Diagrams of Binary Titanium Alloys, J.L. Murray, ed., ASM International, Metals Park, OH, 1987, p 118-122

1989Rud: M.V. Rudometkina, D.Yu. Seropegin, A.V. Gribanov, L.S. Gusei, Phase Equilibria in the Ti-Nb-Ge System at $1170 \mathrm{~K}$, J. Less-Common Met., 1989, 147(2), p 239-247

2011Liu: D. Liu, H. Yan, X. Yuan, Y. Chung, Y. Du, H. Xu, L. Liu, P. Nash, Thermodynamic Modeling of the Ge-Ti System Supported by Key Experiment, Thermochim. Acta, 2011, 521, p $148-154$

Table 1 Ge-Ti crystal structure data

\begin{tabular}{lcclcl}
\hline Phase & Composition, at.\% Ti & Pearson symbol & Space group & Strukturbericht designation & Prototype \\
\hline$(\mathrm{Ge})$ & $0-2.2$ & $c F 8$ & $F d \overline{3} m$ & $A 4$ & $\mathrm{C}(\mathrm{diamond})$ \\
$\mathrm{Ge}_{2} \mathrm{Ti}$ & 33.3 & $o F 24$ & $F d d d$ & $C 54$ & $\ldots$ \\
$\mathrm{Ge}_{5} \mathrm{Ti}_{6}$ & 54.5 & $o I 44$ & $I m m m$ & $D 8_{8}$ & $\mathrm{Nb}_{6} \mathrm{Sn}_{5}$ \\
$\mathrm{Ge}_{3} \mathrm{Ti}_{5}$ & 62.5 & $h P 16$ & $P 6_{3} / m c m$ & $A 2$ & $\mathrm{Mn}_{5} \mathrm{Si}_{3}$ \\
$(\beta \mathrm{Ti})$ & $91.2-100$ & $c I 2$ & $I m \overline{3} m$ & $\mathrm{~A}$ & $\mathrm{Mg}$ \\
$(\alpha \mathrm{Ti})$ & $95.9-100$ & $h P 2$ & $P 6_{3} / m m c$ & & $\mathrm{M}$ \\
\hline
\end{tabular}

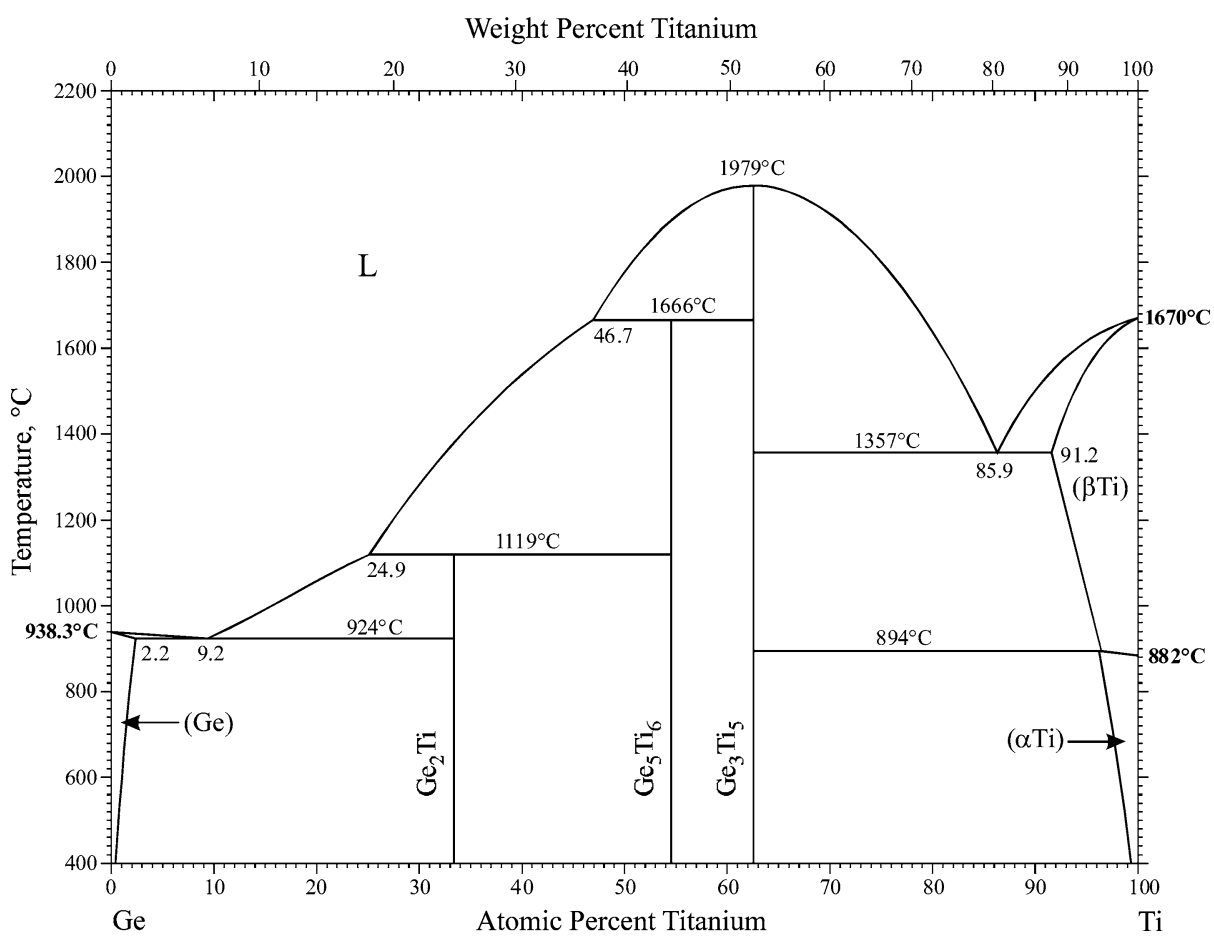

Fig. 1 Ge-Ti phase diagram 